\title{
2-Methoxyestradiol Nanocrystal Colloidal Dispersion
}

National Cancer Institute

\section{Source}

National Cancer Institute. 2-Methoxyestradio/ Nanocrystal Colloidal Dispersion. NCI

Thesaurus. Code C62603.

An orally bioavailable liquid formulation containing the small molecule 2-methoxyestradiol with potential antineoplastic activity. 2-Methoxyestradiol, a naturally occurring estradiol metabolite, exerts its antitumor effect by inhibiting endothelial cells as well as tumor cells through multiple mechanisms. This agent binds to tubulin and disrupts microtubule formation, thereby preventing mitosis and subsequent cellular proliferation. In addition, 2-methoxyestradiol induces caspase activation, resulting in cell cycle arrest in G2 phase and apoptosis in due course. This agent also down-regulates hypoxia inducible factorone alpha (HIF-1a). The nanocrystal colloidal dispersion increases the absorption of 2methoxyestradiol, thereby improving its bioavailability, which results in enhanced drug plasma levels. 